\title{
Commentary
}

\section{Macroscopic Quantum Coherence in Patient-Practitioner-Remedy Entanglement: The Quantized Fluctuation Field Perspective}

\author{
Alex Hankey \\ SVYASA, Jigani, Bangalore, India
}

Keywords: critical instabilities - macroscopic wave functions - PPR entanglement - quantized fluctuations - quantum coherence - vibrational medicine

\section{Objections to Patient-Practitioner-Remedy Entanglement}

Lionel Milgrom's article (1) has stirred up considerable correspondence (2), mostly due to misconceptions of various kinds. Chrastina, for example (3), is quite wrong to attack Milgrom's work over whether coherence in quantum systems can be maintained over macroscopic distances at high temperatures. All quantum field theories in solid state physics provide examples where this kind of assumption is made at a primal level, since the low energy forms of their various quanta are assumed to extend over the whole lattice being considered. Theoretically that is infinite in thermodynamic systems, and, practically, over a whole crystal, or whatever kind of domain is appropriate to the exciton under consideration, be it phonon, electron, magnon or other. The Mossbauer effect (4) provides a fine example of how a macroscopic system can undergo a quantum interaction as a coherent whole at whatever temperature it may happen to find itself.

In the case of conscious systems, macroscopic quantum coherence as a fundamental requirement was first hypothesized by Domash (5) working in close conjunction with Maharishi Mahesh Yogi in the 1970s. The two used it to make highly successful predictions about brain states in higher states of consciousness (6), which have been greatly extended over the years $(7,8)$. Their work has been taken up and championed by Penrose (9), who also insists that macroscopic quantum coherence has to be a major factor in allowing the brain to maintain conscious awareness (10, p.133) of non-computational

For reprints and all correspondence: Alex Hankey, SVYASA, Jigani, Bangalore, India. E-mail: alexhankey@gmail.com kinds he has so brilliantly established. Penrose also recognizes that such brain states must be inherently weakly interacting so that coherence can be maintained $(10$, p.134).

\section{Instability Fluctuations}

My own work has developed these ideas along a different line (11), which relates directly to homeopathy, and entirely vindicates Milgrom's assumptions. It is based on a neglected area of physics - the quantum properties of instability fluctuations. It can be shown that fluctuations at feedback instabilities provide a realistic model of the previously rejected concept of the life force (12). I developed this specifically out of a biophysical model for the action of vibrational medicines (11), including ultra-diluted ones: simple assumptions about toxin action on enzyme active sites, coupled with the role of fluctuations in preventing regulatory switching failures and consequent maladaptation, permit the homeopathic principle to be derived in the context of the model (11).

All biological control systems have to contain feedback instabilities/critical points; these occur in cellular regulation of gene expression (12), and also in neural networks (13). The latter means that quantized fluctuation fields apply to brain states hypothesized to support conscious awareness, as well as to the vital force at the cellular level. Recently, I have confirmed the correctness of the application of quantized fluctuation fields through a completely different process of reasoning: such fields are effectively in states of self-observation (14), they provide an information space of the 'dual' kind proposed by David Chalmers as a fundamental requirement in his non-reductive approach to modeling self-conscious 
awareness (15). Since they also yield models of vibrational medicines used to stimulate the vital force's curative action (11), such fields do not simply validly represent states of patient, practitioner and remedy, their use is mandatory in any attempt to do so.

\section{Macroscopic Quantum Coherence at Instabilities}

Now for the crunch: in their quantum form, feedback instability fluctuations exhibit macroscopic coherence. Why? Because, at critical instabilities, many thermodynamic functions diverge, including the specific heat, and the correlation length, and, as we shall see, the effect of the first divergence is to create quantum coherence, while the second makes that coherence macroscopic. Divergence of specific heat at critical points was theoretically predicted by Onsager in his famous solution of the two dimensional Ising model (16); it has been much observed experimentally, e.g. at the gas-liquid critical point of Argon (17), for which it diverges logarithmically i.e. exponent $\alpha=0$ (Fig. 1), though in magnetic systems $\alpha>0$ (18). Divergence of the correlation length was predicted by Fisher and Burford (19) for Ising Models, and by Ritchie and Fisher (20) for more complex magnetic models. Experimentally, it is always found to diverge e.g. for gas-liquid critical phenomena in Xenon by Canell and Benedek (21), and for binary liquid mixtures by Berge et al. (22). Such critical point divergences are quite general.

To understand why these results imply macroscopic quantum coherence at critical points, we must remember that any unique dynamics of critical points must result from particular properties of the system of excitations that are unique to critical points. That means from the fluctuations, and, in terms of atomic physics, from their quantum form. Thus the anomalous, divergent behavior of the specific heat and the correlation length can only result from specific properties of the (quantized form of the) critical point fluctuations. As the temperature $T$ approaches the critical temperature $T_{\mathrm{c}}$ from above, the increase in specific heat $[(16,17)$, Fig. 1] means that extra heat energy is lost; implying that systems of critical fluctuations have anomalously low energy/heat content. This translates into low entropy content, since, by $d q=T d S$, heat change $d q$ is directly related to entropy change $d S$. For an ensemble of quantum systems, entropy decreases as coherence in its density matrix increases. In such systems, the only way for anomalous quantum entropy decrease to occur is by increasing the overall quantum coherence: the specific excitons, which become coherent, must be the quantized instability fluctuations.

Similarly, divergence of system correlation length at critical (instability) points (16-19), must specifically refer to the correlation length associated with the fluctuations (13).

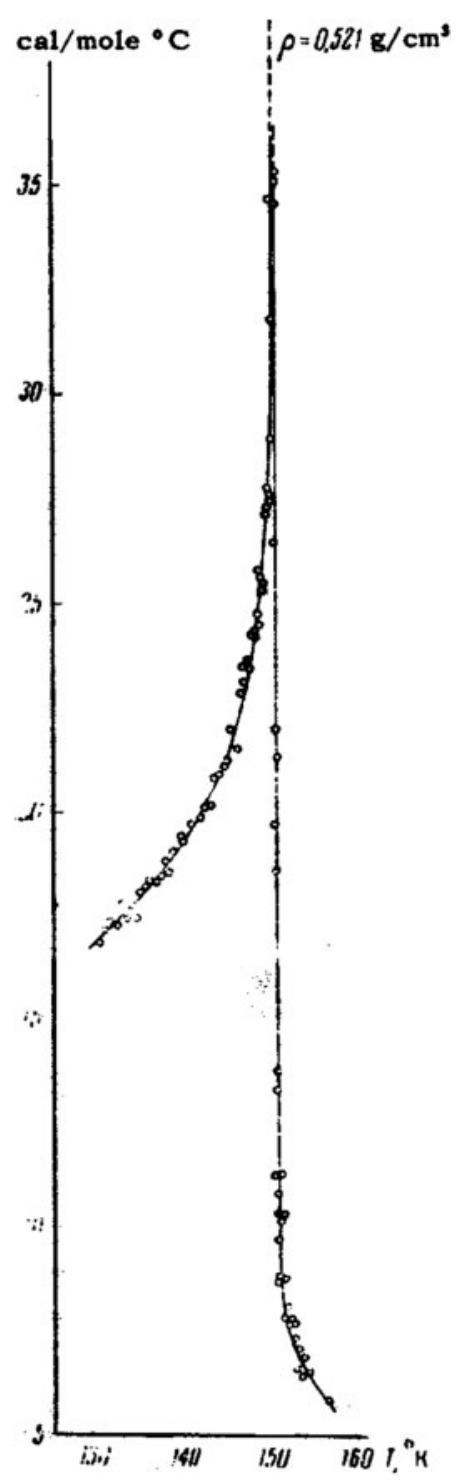

Figure 1. The divergence of specific heat at critical instabilities. This graph presents data on the specific heat of the gas Argon at its gasliquid critical point. The specific heat becomes larger and larger as the critical temperature is approached. By plotting the specific heat against the logarithm of the temperature difference with the critical temperature, the logarithmic nature of the divergence is made clear. At a quantum level, such anomalous changes in heat capacity and content can only be taken up by coherence based entropy changes.

But since the fluctuations gain anomalously large levels of quantum coherence, and since coherence means correlations, the divergence of their correlation length can only mean that the correlation length of their quantum coherence must be divergent. A divergent correlation length means that the quantum coherence's originally microscopic correlation length now becomes macroscopic. We conclude that, generally, at all critical instabilities (including feedback instabilities in biological regulatory systems), quantum fluctuation fields exhibit macroscopic quantum coherence. 


\section{Conclusions}

The property of macroscopic quantum coherence at critical points impacts many fields. The arguments provided for it here are macroscopic and heuristic. In further publications, we shall explore its implications for critical phenomena in general, and quantization of anharmonic oscillators in particular, in order to provide the theory with a microscopic foundation. At this stage, we can draw three conclusions.

First, Milgrom's assumption of macroscopic quantum coherence (1) applies to quantum fluctuation fields of all kinds: those that represent the vital force/consciousnesses of the practitioner and patient (14), and those representing the homeopathic remedy (11). His assumptions are therefore completely justified, provided that his wave functions are taken to be those of quantized instability fluctuations, which they have to be, because those alone satisfy Chalmers's 'Dual Aspect' condition $(14,15)$.

Second, quantized fluctuation fields on neural networks only interact weakly with other physical fields; they satisfy Penrose's second requirement (10, p.134). Entanglement between such states will not be subject to decoherence effects, and will be long-lived. In principle objections (3) are invalid. With these provisos, the work of Milgrom $(23,24)$ and those with related outlooks $(25)$ should be recognized for what it is: pioneering physics of great originality, with tremendous scope for further development (26).

Finally, this work supports the Domash (5) and Penrose et al. (10, p.133) hypothesis that macroscopic quantum coherence exists in brain states of conscious awareness. This represents a vital, additional property of quantized instability fluctations. It enables them to fulfill another stringent requirement for models of consciousness laid down by previous theory.

\section{References}

1. Milgrom LR. Journeys in the country of the blind: entanglement theory and the effects of blinding on trials of homeopathy and homeopathic provings. eCAM 2007;4:7-16.

2. Baker SJ. Re: Homeopathy and Hubris. eLetters. eCAM December 27, 2007.

3. Chrastina D. Re: Response to Simon Baker. eLetters. eCAM November 6, 2007.

4. Mössbauer RL. Gammastrahlung in Ir191. Z. Physik 1958;151:124.

5. Domash L. The transcendental meditation technique and quantum physics: is pure consciousness a macroscopic quantum state in the brain? Paper 99. In: Orme-Johnson DJ and Farrow JT (eds). Scientific Research on the Transcendental Meditation Program. Collected Papers. Volume 1. Rheinweiler, MERU Press, 1977.
6. Orme-Johnson DJ. The Dawn of the Age of Enlightenment: experimental evidence that the transcendental meditation technique produces fourth and fifth states of consciousness in the individual and a profound influence of orderliness in society. Paper 100. In: Orme-Johnson DJ and Farrow JT (eds). Scientific Research on the Transcendental Meditation Program. Collected Papers. Volume 1. Rheinwiler, MERU Press, 1977.

7. Travis F, Pearson C. Pure consciousness: distinct phenomenological and physiological correlates of 'consciousness itself'. Int $J$ Neurosci 2000;100:1/4.

8. Travis F, Tecce J, Arenander A, Wallace RK. Patterns of EEG coherence, power, and contingent negative variation characterize the integration of transcendental and waking states. Biol Psychol 2002;61:293/319.

9. Penrose R. The Emperor's New Mind. Oxford: Oxford University Press, 1989

10. Penrose R, Hawking S, Cartwright N, Shimony A. The Large, the Small, and the Human Mind, Vol. 133. Cambridge: Cambridge University Press, 2007.

11. Hankey A. Are we close to a theory of energy medicine? J Alt Comp Med 2004;10:83-6.

12. Hankey A. CAM modalities can stimulate advances in theoretical biology. Evid Based Comp Alt Med (eCAM) 2005;2:5-12.

13. Torres J-L. Homeopathic effect: a network perspective. Homeopathy 2002;91:89-94.

14. Hankey A. New physics models of conscious experience: Quantum singularities and the mind-matter and mind-body problems. Int $J$ Yoga (I-JOY) to be published.

15. Chalmers D. Facing up to the problem of consciousness. In: Shear J. (ed). Explaining Consciousness: the Hard Problem. Cambridge, Mass: MIT Press, 1997.

16. Onsager L. Crystal Statistics: I. a two-dimensional model with an order-disorder transition. Phys Rev 1944;65:117-49.

17. Bagatskii MI, Voronel AV, Gusak VG. Measurement of the specific heat $\mathrm{C}_{\mathrm{v}}$ of argon in the immediate vicinity of the critical point. Soviet Phys JETP 1963;16:517-8.

18. Stanley HE. Introduction to Phase Transitions and Critical Phenomena. London: Oxford University Press, 1971.

19. Fisher ME, Burford RJ. Theory of critical point scattering and correlations I. The Ising Model. Phys Rev 1967;156:583-622.

20. Ritchie DS, Fisher ME. Theory of critical point scattering and correlations II. Heisenberg Models. Phys Rev 1972; B5:2668-92.

21. Canell DS, Benedek GB. Brillouin spectrum of Xenonnear its critical point. Phys Rev Lett 1970;25:1157-61.

22. Berge P, Calmettes P, Laj C, Tournarie M, Volochine B. Dynamics of concentration fluctuations in a binary mixture in the hydrodynamical and non-hdrodynamical Regimes. Phys Rev Lett 1970;24:1223-5.

23. Milgrom LR. Patient-practitioner-remedy entanglement: part 7. A gyroscopic metaphor for the vital force and its use to illustrate some of the empirical laws of homeopathy. Forsch Komplementarmed Klass Naturheilkd 2004;11:212-23.

24. Milgrom LR. Patient-practitioner-remedy (PPR) entanglement, Part 10: toward a unified theory of homeopathy and conventional medicine. J Altern Complement Med 2007;13:759-70.

25. Walach H. Generalised entanglement: a new theoretical model for understanding the effects of complementary an alternative medicine. J Altern Complement Med 2005;11:549-59.

26. Hankey A. Self Consistent Theories of Health and Healing. $J$ Altern Complement Med 2008;14:221-223.

Received March 27, 2008; accepted April 4, 2008 


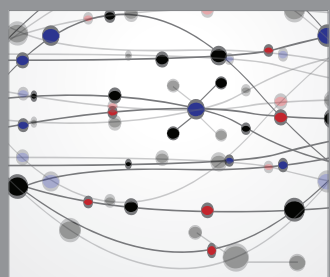

The Scientific World Journal
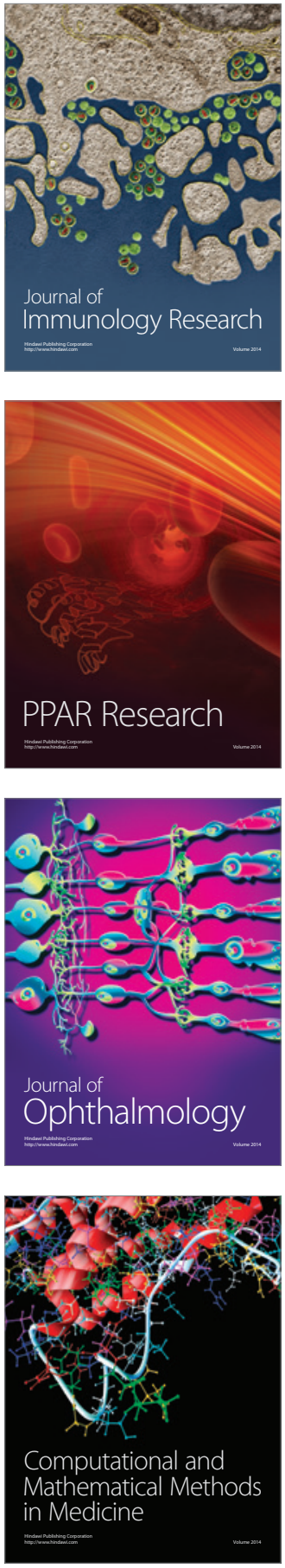

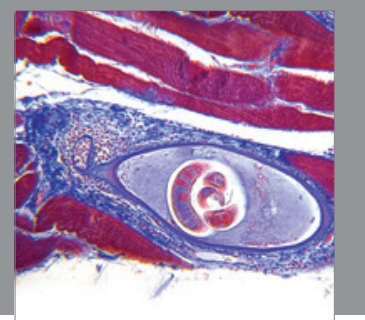

Gastroenterology

Research and Practice
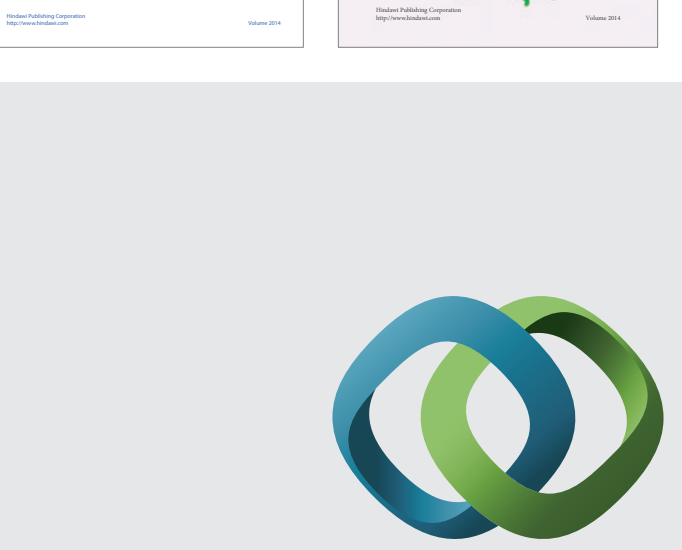

\section{Hindawi}

Submit your manuscripts at

http://www.hindawi.com
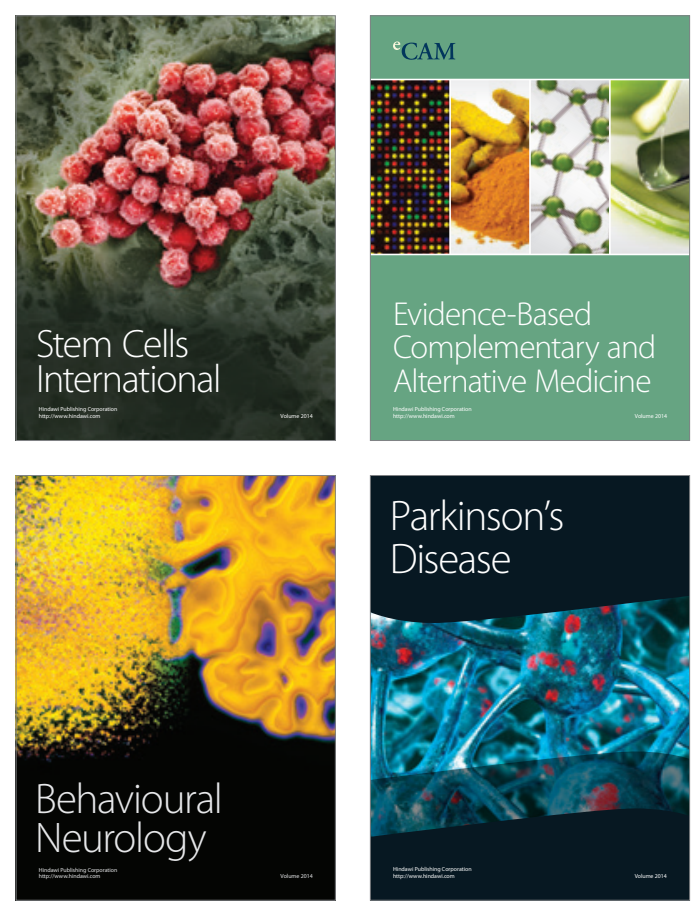

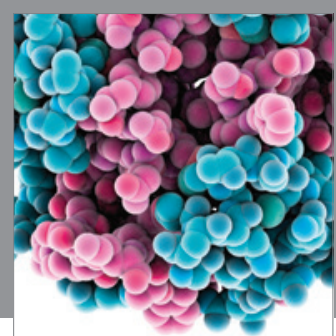

Journal of
Diabetes Research

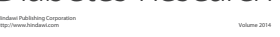

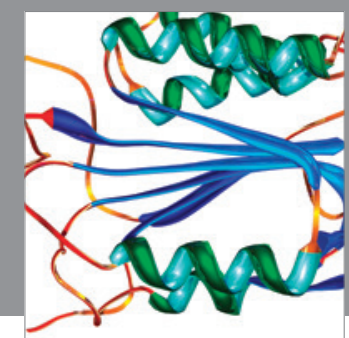

Disease Markers
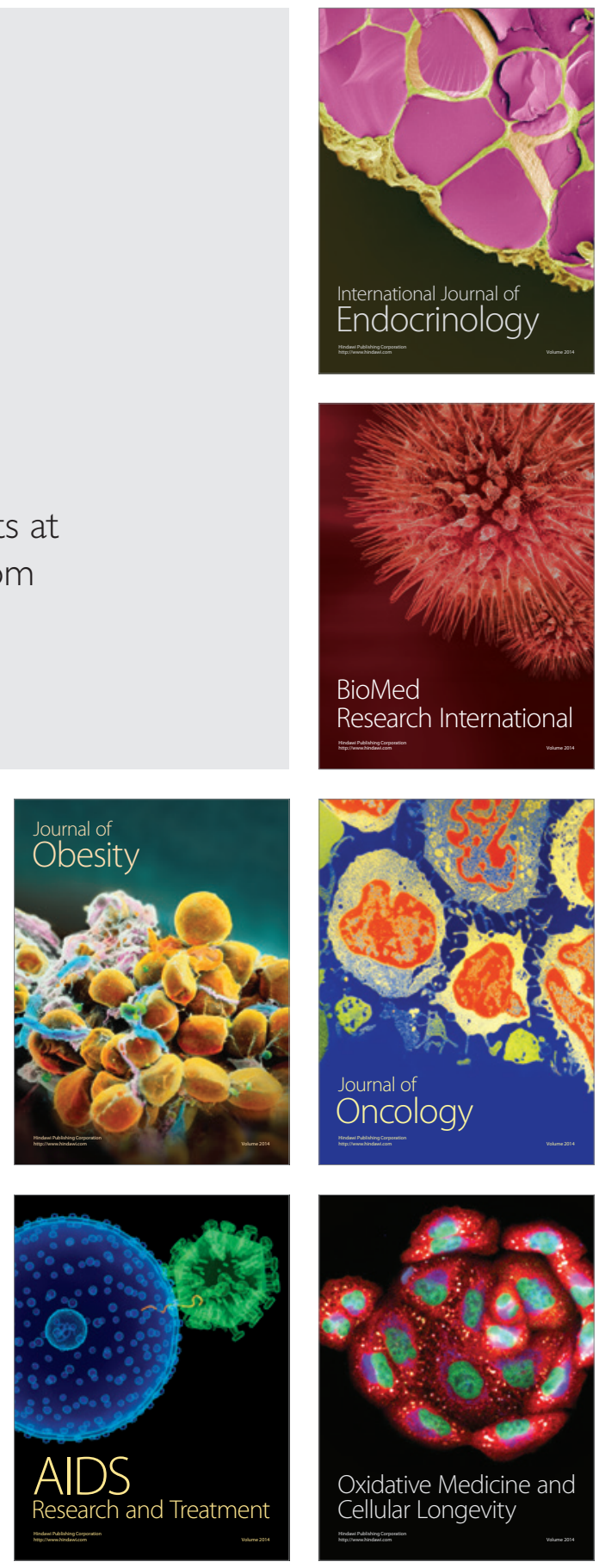